Elisabetta Tornatore and Stefania Maria Buccellato (Palermo)

\title{
ON A STOCHASTIC SIR MODEL
}

Abstract. We consider a stochastic SIR system and we prove the existence, uniqueness and positivity of solution. Moreover the existence of an invariant measure under a suitable condition on the coefficients is studied.

1. Introduction. In [15] we analyzed the stability of the disease free equilibrium of the stochastic SIR model

$$
\left\{\begin{array}{l}
d S(t)=(\mu-\beta S(t) I(t)-\mu S(t)) d t-\sigma S(t) I(t) d W(t), \\
d I(t)=(\beta S(t) I(t)-(\lambda+\mu) I(t)) d t+\sigma S(t) I(t) d W(t), \\
d R(t)=(\lambda I(t)-\mu R(t)) d t
\end{array}\right.
$$

where $S(t), I(t)$ and $R(t)$ denote the number of individuals susceptible to the disease, infected and removed from the possibility of infection through full immunity, respectively. The constant $\beta$ is the contact rate that represents the average number of contacts per infective per day, $\lambda$ is the recovery rate of infected people, $\mu$ is the death and birth rate, $\sigma$ is a positive constant and $W(t)$ is a real Wiener process defined on a stochastic basis $\left(\Omega, \mathcal{F},\left(\mathcal{F}_{t}\right)_{t \geq 0}, \mathbb{P}\right)$. We suppose that all newborns are susceptible and, of course, $\mu, \lambda, \sigma, \beta \in \mathbb{R}_{+}$.

In particular, by using a suitable Lyapunov function, we proved the stability of the disease free equilibrium $E_{0}=(1,0,0)$ of the system (1) under the condition $0<\beta<\min \left\{\mu+\lambda-\sigma^{2} / 2,2 \mu\right\}$. A numerical simulation showed that the disease free equilibrium $E_{0}$ is stable also if $\min \left\{\mu+\lambda-\sigma^{2} / 2,2 \mu\right\}<$ $\beta<\mu+\lambda+\sigma^{2} / 2$, while if $\beta>\mu+\lambda+\sigma^{2} / 2$ then $E_{0}$ is unstable and the solution of system (1) fluctuates around the endemic equilibrium

$$
E_{+}=\left(\frac{\lambda+\mu}{\beta}, \frac{\mu}{\beta}\left(\frac{\beta}{\lambda+\mu}-1\right), \frac{\lambda}{\beta}\left(\frac{\beta}{\lambda+\mu}-1\right)\right)
$$

of the corresponding deterministic system (see Figure 1).

2000 Mathematics Subject Classification: 60H10, 92D30.

Key words and phrases: stochastic process, invariant measure, disease models. 

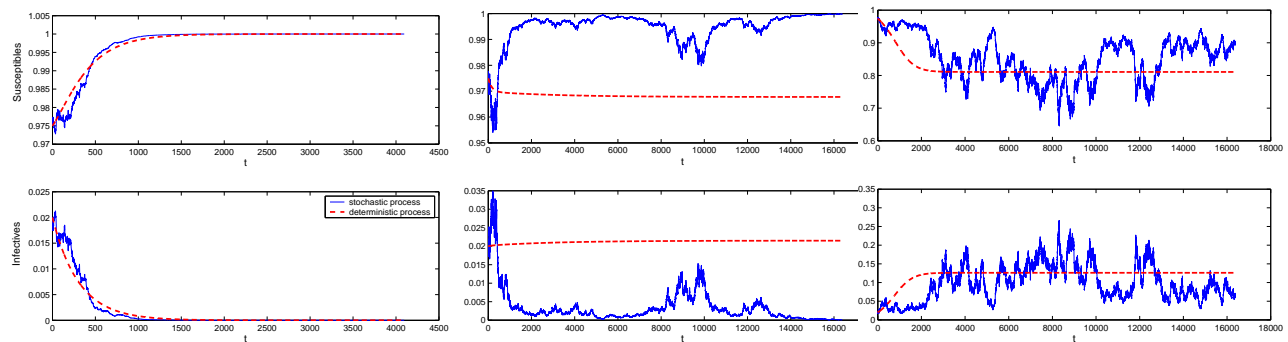

Fig. 1. Number of susceptible and infected individuals in the deterministic and stochastic SIR model when: $\lambda=0,1, \mu=0,2, \sigma=0,15, \beta=0,2\left(0<\beta<\min \left\{\mu+\lambda-\sigma^{2} / 2,2 \mu\right\}\right)$; $\lambda=0,1, \mu=0,2, \sigma=0,2, \beta=0,31\left(\min \left\{\mu+\lambda-\sigma^{2} / 2,2 \mu\right\}<\beta<\mu+\lambda+\sigma^{2} / 2\right)$ and $\lambda=0,1, \mu=0,2, \sigma=0,2, \beta=0,37\left(\beta>\mu+\lambda+\sigma^{2} / 2\right)$ respectively

In the present paper we will prove the existence, uniqueness and positivity of solution of the system (1) (see Figure 2). Furthermore, under the condition $\beta>\mu+\lambda+\sigma^{2} / 2$ we will prove the existence and uniqueness of the invariant measure.

2. Existence and uniqueness of solution. In order to prove the existence, uniqueness and positivity of solution of the system (1) we introduce the notation

$$
\mathbb{R}_{+}^{3}=\left\{x \in \mathbb{R}^{3}: x_{i}>0 \text { for all } i=1,2,3\right\}
$$

and we study system (1) with initial conditions

$$
(S(0), I(0), R(0)) \in \mathbb{R}_{+}^{3}, \quad S(0)+I(0)+R(0)=1 .
$$

Since the coefficients of the system (1) are locally Lipschitz the following result on the local existence of solutions holds:

Theorem 2.1 ([4, Theorem 1.1, p. 98]). For any given initial condition $(S(0), I(0), R(0)) \in \mathbb{R}_{+}^{3}$ there exists $\tau>0$ and a unique solution $(S(t), I(t), R(t))$ to the system (1) for $t \in[0, \tau)$.

By using (2) we can consider an integer $k_{0}>2$ sufficiently large such that

$$
(S(0), I(0), R(0)) \in\left[1 / k_{0}, 1-1 / k_{0}\right]^{3} .
$$

For each integer $k>k_{0}$ we define the stopping time

$$
\tau_{k}(\omega)=\inf \left\{t \in[0, \tau):(S(t, \omega), I(t, \omega), R(t, \omega)) \notin[1 / k, 1-1 / k]^{3}\right\}
$$

where we set $\inf \emptyset=\infty$. We shall show that the solution of (1) satisfying (2) is positive and global using the idea of [9] (see also [3]).

TheOREM 2.2. There exists a unique solution $(S(t), I(t), R(t))$ to the system (1) with the condition (2) for all $t \geq 0$, and the solution remains in $\mathbb{R}_{+}^{3}$ with probability 1 , that is,$(S(t), I(t), R(t)) \in \mathbb{R}_{+}^{3}$ for all $t \geq 0$ a.s. 
For the proof of the theorem we need the following lemma.

Lemma 2.1. Let $(S(t), I(t), R(t))$ be the solution of the system (1) with the condition (2). Then

(4) $E\left(\log ^{2} S\left(\tau_{k}(\omega) \wedge t\right)+\log ^{2} I\left(\tau_{k}(\omega) \wedge t\right)+\log ^{2} R\left(\tau_{k}(\omega) \wedge t\right)\right) \leq C(t)$

where $C(t)$ is the solution of the Cauchy problem

$$
\left\{\begin{array}{l}
y^{\prime}(t)=3 \beta+9 \mu+3 \lambda+5 \sigma^{2}+\left(\beta+3 \mu+\lambda+\sigma^{2}\right) y(t), \\
y(0)=\log ^{2} S(0)+\log ^{2} I(0)+\log ^{2} R(0) .
\end{array}\right.
$$

Proof. We consider the $C^{2}$-function $\Upsilon: \mathbb{R}_{+}^{3} \rightarrow \mathbb{R}_{+}$defined by

$$
\Upsilon\left(x_{1}, x_{2}, x_{3}\right)=\log ^{2} x_{1}+\log ^{2} x_{2}+\log ^{2} x_{3} .
$$

If $(S(t), I(t), R(t)) \in \mathbb{R}_{+}^{3}$ the Ito formula shows that

$$
\begin{aligned}
d\left(\log ^{2} S(t)\right. & \left.+\log ^{2} I(t)+\log ^{2} R(t)\right)=2\left[\left(\frac{\mu}{S}-\beta I(t)-\mu-\frac{\sigma^{2}}{2} I^{2}(t)\right) \log S(t)\right. \\
+ & \left(\beta S(t)-(\lambda+\mu)-\frac{\sigma^{2}}{2} S^{2}(t)\right) \log I(t)+\left(\lambda \frac{I(t)}{R(t)}-\mu\right) \log R(t) \\
+ & \left.\frac{\sigma^{2}}{2}\left(I^{2}(t)+S^{2}(t)\right)\right] d t+2 \sigma(-I(t) \log S(t)+S(t) \log I(t)) d W
\end{aligned}
$$

In virtue of the definition (3), for all $t<\tau_{k}(\omega)$ we have $(S(t), I(t), R(t)) \in$ $[1 / k, 1-1 / k]^{3}$, so

$$
\begin{aligned}
\log ^{2} S\left(\tau_{k}(\omega) \wedge t\right)+\log ^{2} I\left(\tau_{k}(\omega) \wedge t\right)+\log ^{2} R\left(\tau_{k}(\omega) \wedge t\right) \\
=\log ^{2} S(0)+\log ^{2} I(0)+\log ^{2} R(0) \\
\quad+2 \int_{0}^{\tau_{k}(\omega) \wedge t}\left[\left(\frac{\mu}{S\left(t^{\prime}\right)}-\beta I\left(t^{\prime}\right)-\mu-\frac{\sigma^{2}}{2} I^{2}\left(t^{\prime}\right)\right) \log S\left(t^{\prime}\right)\right. \\
\quad+\left(\beta S\left(t^{\prime}\right)-(\lambda+\mu)-\frac{\sigma^{2}}{2} S^{2}\left(t^{\prime}\right)\right) \log I\left(t^{\prime}\right) \\
\left.\quad+\left(\lambda \frac{I\left(t^{\prime}\right)}{R\left(t^{\prime}\right)}-\mu\right) \log R\left(t^{\prime}\right)\right] d t^{\prime}+\sigma^{2} \int_{0}^{\tau_{k}(\omega) \wedge t}\left(I^{2}\left(t^{\prime}\right)+S^{2}\left(t^{\prime}\right)\right) d t^{\prime} \\
\quad+2 \sigma \int_{0}^{\tau_{k}(\omega) \wedge t}\left(-I\left(t^{\prime}\right) \log S\left(t^{\prime}\right)+S\left(t^{\prime}\right) \log I\left(t^{\prime}\right)\right) d W .
\end{aligned}
$$

We neglect the terms

$\int_{0}^{\tau_{k}(\omega) \wedge t} \frac{\mu}{S\left(t^{\prime}\right)} \log S\left(t^{\prime}\right) d t^{\prime}, \quad \int_{0}^{\tau_{k}(\omega) \wedge t} \beta S\left(t^{\prime}\right) \log I\left(t^{\prime}\right) d t^{\prime}, \quad \int_{0}^{\tau_{k}(\omega) \wedge t} \lambda \frac{I\left(t^{\prime}\right)}{R\left(t^{\prime}\right)} \log R\left(t^{\prime}\right) d t^{\prime}$ 
and estimate the terms of the right-hand side by

$$
\begin{aligned}
& \mid 2 \int_{0}^{\tau_{k}(\omega) \wedge t}\left[\left(-\beta I\left(t^{\prime}\right)-\mu-\frac{\sigma^{2}}{2} I^{2}\left(t^{\prime}\right)\right) \log S\left(t^{\prime}\right)\right. \\
& \left.\quad+\left(-(\lambda+\mu)-\frac{\sigma^{2}}{2} S^{2}\left(t^{\prime}\right)\right) \log I\left(t^{\prime}\right)-\mu \log R\left(t^{\prime}\right)\right] d t^{\prime} \mid \\
& \quad \leq 2\left(\beta+3 \mu+\lambda+\sigma^{2}\right) \int_{0}^{\tau_{k}(\omega) \wedge t}\left(\left|\log S\left(t^{\prime}\right)\right|+\left|\log I\left(t^{\prime}\right)\right|+\left|\log R\left(t^{\prime}\right)\right|\right) d t^{\prime}
\end{aligned}
$$

and

$$
\left|\sigma^{2} \int_{0}^{\tau_{k}(\omega) \wedge t}\left(I^{2}\left(t^{\prime}\right)+S^{2}\left(t^{\prime}\right)\right) d t\right| \leq 2 \sigma^{2} t
$$

Substituting these relations into (6) and calculating the mean we have

$$
\begin{aligned}
E\left(\log ^{2}\right. & \left.S\left(\tau_{k}(\omega) \wedge t\right)+\log ^{2} I\left(\tau_{k}(\omega) \wedge t\right)+\log ^{2} R\left(\tau_{k}(\omega) \wedge t\right)\right) \\
\leq & \log ^{2} S(0)+\log ^{2} I(0)+\log ^{2} R(0) \\
& +2 \sigma^{2} t+2\left(\beta+3 \mu+\lambda+\sigma^{2}\right) \\
& \times \int_{0}^{t} E\left(\left|\log S\left(\tau_{k}(\omega) \wedge t^{\prime}\right)\right|+\left|\log I\left(\tau_{k}(\omega) \wedge t^{\prime}\right)\right|+\left|\log R\left(\tau_{k}(\omega) \wedge t^{\prime}\right)\right|\right) d t^{\prime} .
\end{aligned}
$$

By the Hölder inequality,

$$
\begin{aligned}
& E\left(\left|\log S\left(\tau_{k}(\omega) \wedge t^{\prime}\right)\right|+\left|\log I\left(\tau_{k}(\omega) \wedge t^{\prime}\right)\right|+\left|\log R\left(\tau_{k}(\omega) \wedge t^{\prime}\right)\right|\right) \\
& \quad \leq \frac{3}{2}+\frac{1}{2} E\left(\log ^{2} S\left(\tau_{k}(\omega) \wedge t^{\prime}\right)+\log ^{2} I\left(\tau_{k}(\omega) \wedge t^{\prime}\right)+\log ^{2} R\left(\tau_{k}(\omega) \wedge t^{\prime}\right)\right) .
\end{aligned}
$$

Substituting we obtain

$$
\begin{aligned}
& E\left(\log ^{2} S\left(\tau_{k}(\omega) \wedge t\right)+\log ^{2} I\left(\tau_{k}(\omega) \wedge t\right)+\log ^{2} R\left(\tau_{k}(\omega) \wedge t\right)\right) \\
& \leq \log ^{2} S(0)+\log ^{2} I(0)+\log ^{2} R(0)+\left(3 \beta+9 \mu+3 \lambda+5 \sigma^{2}\right) t \\
& \quad+\left(\beta+3 \mu+\lambda+\sigma^{2}\right) \\
& \quad \times \int_{0}^{t} E\left(\log ^{2} S\left(\tau_{k}(\omega) \wedge t^{\prime}\right)+\log ^{2} I\left(\tau_{k}(\omega) \wedge t^{\prime}\right)+\log ^{2} R\left(\tau_{k}(\omega) \wedge t^{\prime}\right)\right) d t^{\prime} .
\end{aligned}
$$

We set

$$
Y(t)=E\left(\log ^{2} S\left(\tau_{k}(\omega) \wedge t\right)+\log ^{2} I\left(\tau_{k}(\omega) \wedge t\right)+\log ^{2} R\left(\tau_{k}(\omega) \wedge t\right)\right) ;
$$

taking into account this relation, from (7) we get

$$
Y(t) \leq Y(0)+\left(3 \beta+9 \mu+3 \lambda+5 \sigma^{2}\right) t+\left(\beta+3 \mu+\lambda+\sigma^{2}\right) \int_{0}^{t} Y\left(t^{\prime}\right) d t^{\prime},
$$


from which it follows that

$$
Y(t) \leq C(t), \quad \forall t \geq 0,
$$

where $C(t)$ is the solution of the Cauchy problem in the statement of the lemma.

Proof of Theorem 2.2. By Theorem 2.1 there exist $\tau>0$ and the solution $(S(t), I(t), R(t))$ to the system (1) with the condition (2) for $t \in[0, \tau)$; to prove that this solution is global, we need to show that $\tau=\infty$ a.s. Consider the stopping time defined in (3). Clearly $\left(\tau_{k}\right)$ is an increasing sequence. Set $\tau_{\infty}(\omega)=\lim _{k \rightarrow \infty} \tau_{k}(\omega)$, whence $\tau_{\infty}(\omega)<\tau$ a.s. If we can show that $\tau_{\infty}(\omega)=\infty$ a.s. then $\tau=\infty$ a.s. and consequently $(S(t), I(t), R(t)) \in \mathbb{R}_{+}^{3}$ for all $t \geq 0$ a.s. If this statement is false, then there are a constant $T>0$ and $\varepsilon \in(0,1)$ such that

$$
\mathbb{P}\left\{\omega \in \Omega: \tau_{\infty}(\omega) \leq T\right\}>\varepsilon .
$$

Consequently, there exists an integer $k_{1} \geq k_{0}$ such that

$$
\mathbb{P}\left\{\omega \in \Omega: \tau_{k}(\omega) \leq T\right\} \geq \varepsilon \quad \forall k \geq k_{1} .
$$

Set $\Omega_{k}=\left\{\omega \in \Omega: \tau_{k}(\omega) \leq T\right\}$ for each $k \geq k_{1}$; we have $\mathbb{P}\left(\Omega_{k}\right) \geq \varepsilon$. Note that for every $\omega \in \Omega_{k}$ some component of $\left(S\left(\tau_{k}(\omega)\right), I\left(\tau_{k}(\omega)\right), R\left(\tau_{k}(\omega)\right)\right)$ equals $1 / k$ or $1-1 / k$, and hence by $(6)$,

$$
\Upsilon\left(\left(S\left(\tau_{k}(\omega)\right), I\left(\tau_{k}(\omega)\right), R\left(\tau_{k}(\omega)\right)\right), \omega\right) \geq \log ^{2} k .
$$

From (4) we deduce that

$$
C(T) \geq E\left(1_{\Omega_{k}} \Upsilon\left(\left(S\left(\tau_{k}(\omega)\right), I\left(\tau_{k}(\omega)\right), R\left(\tau_{k}(\omega)\right)\right), \omega\right)\right) \geq \varepsilon \log ^{2} k
$$

where $1_{\Omega_{k}}$ is the indicator function of $\Omega_{k}$. Letting $k \rightarrow \infty$ leads to the contradiction

$$
\infty=C(T)<\infty
$$

so we must have $\tau=\infty$ a.s.

Theorem 2.2 and Lemma 2.1 show that $Q=[0,1] \times[0,1] \times[0,1]$ is the invariant set of the solutions of the system (1).
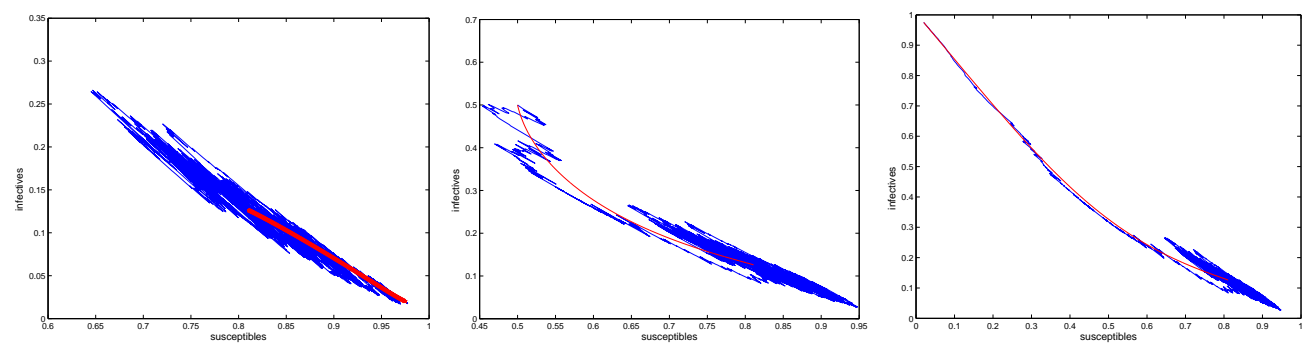

Fig. 2. Susceptibles-infectives phase plane in the deterministic and stochastic SIR model when $\lambda=0,1, \mu=0,2, \sigma=0,2, \beta=0,37\left(\beta>\mu+\lambda+\sigma^{2} / 2\right)$ with different initial data 
3. Existence of the invariant measure under the condition $\beta>$ $\lambda+\mu+\sigma^{2} / 2$. From the system (1) we find that $S(t)+I(t)+R(t)=1$ a.s. for all $t \geq 0$ so we can consider only the first two equations of (1). Moreover, by using the existence, uniqueness and positivity of solution we substitute

$$
\xi(t)=\log S(t), \quad \eta(t)=\log I(t),
$$

and we replace (1) by

$$
\left\{\begin{array}{c}
d \xi=\left(-\beta e^{\eta}-\mu+\mu e^{-\xi}-\frac{\sigma^{2}}{2} e^{2 \eta}\right) d t-\sigma e^{\eta} d W, \\
d \eta=\left(\beta e^{\xi}-(\lambda+\mu)-\frac{\sigma^{2}}{2} e^{2 \xi}\right) d t+\sigma e^{\xi} d W, \\
\xi_{0}=\log S(0), \quad \eta_{0}=\log I(0) .
\end{array}\right.
$$

The aim of this section is to study the existence and uniqueness of the invariant measure of the system (8) by showing that there exists a Has'minskij function (see [4, Chap. III]).

We say that a non-negative function $V \in C^{2}\left(\mathbb{R}^{n}\right)$ is a Has'minskij function for a stochastic equation in $\mathbb{R}^{n}$

$$
d X(t)=b(X(t)) d t+\sigma(X(t)) d W(t)
$$

where $W(t)$ is a Brownian motion in $\mathbb{R}^{n}$, if

$$
\sup _{|x| \geq r} A V(x) \rightarrow-\infty \quad \text { as } r \rightarrow+\infty,
$$

where $A$ is the operator defined by

$$
A v=\frac{1}{2} \sum_{i, j=1}^{n} a_{i j}(x) \frac{\partial^{2} v}{\partial x_{i} \partial x_{j}}+\sum_{i=1}^{n} b_{i}(x) \frac{\partial v}{\partial x_{i}}, \quad a_{i j}(x)=\sum_{k=1}^{n} \sigma_{i k}(x) \sigma_{j k}(x) .
$$

Throughout this section we assume

$$
\beta>\lambda+\mu+\sigma^{2} / 2 .
$$

The operator $A$ for the system (8) is of the form

$$
\begin{aligned}
& \text { 13) } A v=\left(-\beta e^{\eta}-\mu+\mu e^{-\xi}-\frac{\sigma^{2}}{2} e^{2 \eta}\right) \frac{\partial v}{\partial \xi} \\
& +\left(\beta e^{\xi}-(\mu+\lambda)-\frac{\sigma^{2}}{2} e^{2 \xi}\right) \frac{\partial v}{\partial \eta}+\frac{1}{2} \sigma^{2}\left(e^{2 \eta} \frac{\partial^{2} v}{\partial \xi^{2}}-2 e^{\eta} e^{\xi} \frac{\partial^{2} v}{\partial \xi \partial \eta}+e^{2 \xi} \frac{\partial^{2} v}{\partial \eta^{2}}\right) .
\end{aligned}
$$

We set

$$
\xi^{*}=\inf \left\{\xi<0: \beta e^{\xi}-\mu-\lambda-\frac{\sigma^{2}}{2} e^{2 \xi}>\varepsilon\right\}, \quad \varepsilon=\frac{1}{4}\left(\beta-\mu-\lambda-\frac{\sigma^{2}}{2}\right) .
$$

We observe that, in virtue of $(12), \varepsilon>0$ and moreover $\xi^{*}<0$. 
Lemma 3.1. Let $\varepsilon$ and $\xi^{*}$ be as in (14) and set

$$
k^{*}=\frac{\mu+\lambda+\varepsilon}{\mu\left(e^{-\xi^{*}}-1\right)}, \quad U(\xi, \eta)=-k^{*} \xi-\eta \quad \forall(\xi, \eta) \in Q,
$$

where

$$
Q=\left\{(\xi, \eta) \in \mathbb{R}^{2}: \xi \leq 0, \eta \leq 0\right\} .
$$

Then there exist $\bar{\xi}\left(\leq \xi^{*}\right)$ and $\bar{\eta}<0$ such that

$$
A U(\xi, \eta) \leq-\varepsilon / 2 \quad \forall(\xi, \eta) \in Q \backslash K,
$$

where

$$
K=\left\{(\xi, \eta) \in \mathbb{R}^{2}: \bar{\xi} \leq \xi \leq 0, \bar{\eta} \leq \eta \leq 0\right\} .
$$

Proof. It is easy to prove that

$$
A U=k^{*}\left(\beta e^{\eta}-\mu\left(e^{-\xi}-1\right)+\frac{\sigma^{2}}{2} e^{2 \eta}\right)+\mu+\lambda-\beta e^{\xi}+\frac{\sigma^{2}}{2} e^{2 \xi} .
$$

Since

$$
k^{*} \mu\left(e^{-\xi}-1\right) \rightarrow+\infty \quad \text { as } \xi \rightarrow-\infty
$$

we find $\bar{\xi} \leq \xi^{*}<0$ such that

$$
k^{*}\left(\beta-\mu\left(e^{-\xi}-1\right)+\frac{\sigma^{2}}{2}\right)+\mu+\lambda-\beta e^{\xi}+\frac{\sigma^{2}}{2} e^{2 \xi} \leq-\frac{\varepsilon}{2} \quad \forall \xi \leq \bar{\xi} .
$$

Since

$$
k^{*}\left(\beta e^{\eta}+\frac{\sigma^{2}}{2} e^{2 \eta}\right) \rightarrow 0 \quad \text { as } \eta \rightarrow-\infty
$$

we find $\bar{\eta}<0$ such that

$$
k^{*}\left(\beta e^{\eta}+\frac{\sigma^{2}}{2} e^{2 \eta}\right) \leq \frac{\varepsilon}{2} \quad \forall \eta \leq \bar{\eta} .
$$

We observe that $Q \backslash K$ is the union of the following sets:

$$
\begin{aligned}
& E_{1}=\left\{(\xi, \eta) \in \mathbb{R}^{2}: \xi \leq \bar{\xi}, \eta \leq 0\right\}, \\
& E_{2}=\left\{(\xi, \eta) \in \mathbb{R}^{2}: \bar{\xi} \leq \xi \leq \xi^{*}, \eta \leq \bar{\eta}\right\}, \\
& E_{3}=\left\{(\xi, \eta) \in \mathbb{R}^{2}: \xi^{*} \leq \xi<0, \eta \leq \bar{\eta}\right\} .
\end{aligned}
$$

Taking into account (12), (15), (16), (17) it is easy to verify that $A U \leq-\varepsilon / 2$ on each $E_{i}$.

In order to prove the existence of the invariant measure we use the Has'minskij theorem [7, Th. 5.1] and [16, Lemma 4.1].

THEOREM 3.1. If the relation (12) holds, then the system (8) has an invariant measure on

$$
Q=\left\{(\xi, \eta) \in \mathbb{R}^{2}: \xi \leq 0, \eta \leq 0\right\}
$$


Proof. By using (13) and (15) there exists a constant $C$ such that

$$
\left|\sigma^{2} e^{2 \eta} \frac{\partial^{2}}{\partial \xi^{2}} U^{2}+\sigma^{2} e^{2 \xi} \frac{\partial^{2}}{\partial \eta^{2}} U^{2}-2 \sigma^{2} e^{\xi} e^{\eta} \frac{\partial^{2}}{\partial \xi \partial \eta} U^{2}\right| \leq C
$$

for all $(\xi, \eta) \in Q$. Therefore, as in Lemma 4.1 of [16], we set $V=\frac{1}{2} U^{2}$ and we obtain

$$
\sup _{\xi^{2}+\eta^{2} \geq r} A V(\xi, \eta) \rightarrow-\infty \quad \text { as } r \rightarrow \infty, \xi \leq 0, \eta \leq 0 .
$$

Consequently, as in the proof of the Has'minskij theorem, for all $N>0$ we have

$$
\alpha_{N} \int_{0}^{t} \mathbb{E} \chi_{\left\{\xi^{2}+\eta^{2} \geq N^{2}, \xi \leq 0, \eta \leq 0\right\}} d t^{\prime} \leq \mathbb{E}\left(\xi^{2}(0)+\eta^{2}(0)\right)+t \max \left\{\sup _{(\xi, \eta) \in Q} A V, 0\right\}
$$

where

$$
-\alpha_{N}=\sup \left\{A V: \xi^{2}+\eta^{2} \geq N^{2}, \xi \leq 0, \eta \leq 0\right\} .
$$

Then

$$
\frac{1}{t} \int_{0}^{t} \mathbb{P}\left(\left\{\xi^{2}\left(t^{\prime}\right)+\eta^{2}\left(t^{\prime}\right) \geq N^{2}, \xi \leq 0, \eta \leq 0\right\}\right) d t^{\prime} \leq \frac{C_{1}}{\alpha_{N}}
$$

where $C_{1}$ is a constant independent on $N$. So in virtue of Theorem 2.1 of [7], there exists an invariant measure.

4. Uniqueness of the invariant measure. In this section we prove the uniqueness of the invariant measure and the convergence of the law of $(\xi(t), \eta(t))$ to this measure.

THEOREM 4.1. The system (8) has a unique invariant measure and, as $t \rightarrow \infty$, the solution $(\xi(t), \eta(t))$ converges in law to a random variable $Z_{0}$ in $\mathbb{R}^{2}$ whose law is the invariant measure of (8). Moreover, the support of the invariant measure has the form

$$
E=\left\{(\xi, \eta) \in Q: \theta_{0} \leq e^{\xi}+e^{\eta} \leq 1\right\}
$$

with a certain $\theta_{0}>0$.

In order to prove Theorem 4.1, we need the following lemma:

Lemma 4.1. The transition function $P(t, \xi, \eta, A)\left(t>0,(\xi, \eta) \in \mathbb{R}^{2}\right.$, $A \in B\left(\mathbb{R}^{2}\right)$ ) associated to the system (8) has the density $p\left(t, \xi, \eta, \xi^{\prime}, \eta^{\prime}\right) \in$ $C^{\infty}(] 0, \infty\left[\times \mathbb{R}^{2} \times \mathbb{R}^{2}\right)$.

Proof. Set

$$
X=\sigma\left(e^{\eta} \frac{\partial}{\partial \xi}-e^{\xi} \frac{\partial}{\partial \eta}\right)
$$




$$
\begin{aligned}
Y= & \left(-\beta e^{\eta}-\mu+\mu e^{-\xi}+\frac{\sigma^{2}}{2}\left(e^{\xi+\eta}-e^{2 \eta}\right)\right) \frac{\partial}{\partial \xi} \\
& +\left(\beta e^{\xi}-(\mu+\lambda)+\frac{\sigma^{2}}{2}\left(e^{\xi+\eta}-e^{2 \xi}\right)\right) \frac{\partial}{\partial \eta} .
\end{aligned}
$$

Then the differential operator $A$ defined in (13) can be written as

$$
A=\frac{1}{2} X^{2}+Y \text {. }
$$

We consider the Lie bracket

$$
\begin{aligned}
Z=[X, Y]= & \sigma\left(-\mu e^{\eta-\xi}+(\mu+\lambda) e^{\eta}+\sigma^{2} e^{\xi+2 \eta}\right) \frac{\partial}{\partial \xi} \\
& +\sigma\left(\mu-\mu e^{\xi}-\sigma^{2} e^{2 \xi+\eta}\right) \frac{\partial}{\partial \eta}
\end{aligned}
$$

Now we show that $X$ and $Z$ span $\mathbb{R}^{2}$. In fact, set

$$
\begin{gathered}
\alpha=\sigma e^{\eta}, \quad \beta=-\sigma e^{\xi}, \\
\alpha^{\prime}=\sigma\left(-\mu e^{\eta-\xi}+(\mu+\lambda) e^{\eta}+\sigma^{2} e^{\xi+2 \eta}\right), \\
\beta^{\prime}=\sigma\left(\mu-\mu e^{\xi}-\sigma^{2} e^{2 \xi+\eta}\right) .
\end{gathered}
$$

Then $X$ and $Z$ can be written in the form

$$
X=\alpha \frac{\partial}{\partial \xi}+\beta \frac{\partial}{\partial \eta}, \quad Z=\alpha^{\prime} \frac{\partial}{\partial \xi}+\beta^{\prime} \frac{\partial}{\partial \eta}
$$

and we have

$$
\begin{gathered}
\frac{\alpha^{\prime}}{\alpha}=-\mu e^{-\xi}+\mu+\lambda+\sigma^{2} e^{\xi+\eta}, \quad \frac{\beta^{\prime}}{\beta}=-\mu e^{-\xi}+\mu+\sigma^{2} e^{\xi+\eta}, \\
\frac{\alpha^{\prime}}{\alpha}-\frac{\beta^{\prime}}{\beta}=\lambda \neq 0,
\end{gathered}
$$

So

$$
\frac{\alpha^{\prime}}{\alpha} \neq \frac{\beta^{\prime}}{\beta}
$$

Remembering that (19) holds for all $(\xi, \eta) \in \mathbb{R}^{2}$, in virtue of Theorem 3 of [8] the transition function $P(t, \xi, \eta, A)$ has a density $p\left(t, \xi, \eta, \xi^{\prime}, \eta^{\prime}\right) \in$ $C^{\infty}(] 0, \infty\left[\times \mathbb{R}^{2} \times \mathbb{R}^{2}\right)$.

The invariant measure admits a regular density $\Psi(\xi, \eta)$ such that

$$
\iint_{\mathbb{R}^{2}} \Psi(\xi, \eta) p\left(t, \xi, \eta, \xi^{\prime}, \eta^{\prime}\right) d \xi d \eta=\Psi\left(\xi^{\prime}, \eta^{\prime}\right) \quad \forall t>0,\left(\xi^{\prime}, \eta^{\prime}\right) \in \mathbb{R}^{2} .
$$

To determine the support of the invariant measure we use an idea of [16] to prove the following lemma. 
LEMmA 4.2. Set

$$
\Phi(\theta, \xi)=\frac{\sigma^{2} \theta}{2} e^{2 \xi}+\left(\lambda-\frac{\sigma^{2} \theta^{2}}{2}\right) e^{\xi}-(\lambda+\mu) \theta+\mu .
$$

Then there exists $\left.\theta_{0} \in\right] 0,1[$ such that:

(i) if $0<\theta<\theta_{0}$, then

$$
\Phi(\theta, \xi)>0 \quad \forall \xi<0 ;
$$

(ii) if $\theta_{0}<\theta<1$, then there exist $\xi_{1}, \xi_{2}<0$ such that

$$
\Phi\left(\theta, \xi_{1}\right)>0 \quad \Phi\left(\theta, \xi_{1}\right)>0 ;
$$

(iii) if $\theta \geq 1$, then

$$
\Phi(\theta, \xi)<0 \quad \forall \xi<0 .
$$

Proof. We choose $\theta_{0}=\min \{\sqrt{2 \lambda} / \sigma,(\mu / \lambda+\mu)\}$. Then properties (i)-(iii) follow from elementary calculus.

Proof of Theorem 4.1. In virtue of the definition of $\xi(t)$ and $\eta(t)$, it is clear that the support of any invariant measure is included in

$$
\left\{(\xi, \eta) \in \mathbb{R}^{2}: e^{\xi}+e^{\eta} \leq 1\right\} .
$$

On the other hand, if $(\xi(t), \eta(t))$ is in the region $\left\{e^{\xi}+e^{\eta}<\theta_{0}\right\}$, then in virtue of (i) of Lemma 4.1, we have

$$
\Phi\left(e^{\xi(t)}+e^{\eta(t)}, \xi(t)\right)>0
$$

that is, if we put

$$
\begin{aligned}
& a(\xi, \eta)=a=-\beta e^{\eta(t)}-\mu+\mu e^{-\xi(t)}-\frac{\sigma^{2}}{2} e^{2 \eta(t)} \\
& b(\xi, \eta)=b=\beta e^{\xi(t)}-(\lambda+\mu)-\frac{\sigma^{2}}{2} e^{2 \xi(t)}
\end{aligned}
$$

the angle between the vector $\left(e^{\xi(t)}, e^{\eta(t)}\right)$ and $(a, b)$ is between $-\pi / 2$ and $\pi / 2$.

So (7) can be written

$$
d\left(\begin{array}{l}
\xi \\
\eta
\end{array}\right)=\left(\begin{array}{l}
a \\
b
\end{array}\right) d t+\sigma\left(\begin{array}{c}
-e^{\eta} \\
e^{\xi}
\end{array}\right) d W
$$

This means that, for $t_{1}>t$, we have

$$
e^{\xi\left(t_{1}\right)}+e^{\eta\left(t_{1}\right)}>e^{\xi(t)}+e^{\eta(t)}
$$

which leads, in the same manner as in [14, Lemma 3], to

$$
\mathbb{P}\left\{\theta_{0} \leq e^{\xi(t)}+e^{\eta(t)} \leq 1\right\} \rightarrow 1 .
$$

In order to examine the behavior of $(\xi(t), \eta(t))$ in the region $E$, we consider the family of curves

$$
\left\{\gamma_{\theta}\right\}_{\theta \in\left[\theta_{0}, 1\right]}, \quad \gamma_{\theta}=\left\{(\xi, \eta) \in \mathbb{R}^{2}: e^{\xi}+e^{\eta}=\theta\right\}
$$


If $\theta_{0}<\theta<1$, then in virtue of (ii) of Lemma 4.2 there exist $\left(\xi_{1}, \eta_{1}\right),\left(\xi_{2}, \eta_{2}\right)$ $\in \gamma_{\theta}$ such that

$$
\left(\begin{array}{l}
a \\
b
\end{array}\right) \cdot\left(\begin{array}{c}
e^{\xi_{1}} \\
e^{\eta_{1}}
\end{array}\right)>0, \quad\left(\begin{array}{l}
a \\
b
\end{array}\right) \cdot\left(\begin{array}{l}
e^{\xi_{2}} \\
e^{\eta_{2}}
\end{array}\right)<0 .
$$

On the other hand, we can construct the oriented curve $\delta_{(\xi, \eta)}$ which is the trajectory of the differential equation

$$
\begin{aligned}
\frac{d \bar{\xi}(s)}{d s} & =a(\bar{\xi}(s), \bar{\eta}(s)), & & \frac{d \bar{\eta}(s)}{d s}=b(\bar{\xi}(s), \bar{\eta}(s)), \\
\bar{\xi}(0) & =\xi, & \bar{\eta}(0) & =\eta .
\end{aligned}
$$

From Lemma 4.2 it follows that $\delta_{(\xi, \eta)}$ with $(\xi, \eta) \in E$ cannot go out of $E$. Remembering the regularity of the function $\Phi(\theta, \xi)$, it is easy to see that (21) and the construction of $\delta_{(\xi, \eta)}$ imply that, from an arbitrary point $\left(\xi_{1}, \eta_{1}\right)$ such that $\theta_{0}<e^{\xi_{1}}+e^{\eta_{1}}<1$ to an arbitrary point $\left(\xi_{2}, \eta_{2}\right)$ such that $\theta_{0}<e^{\xi_{2}}+e^{\eta_{2}}<1$ we can construct a curve which follows $\gamma_{\theta}, \theta_{0}<\theta<1$, or $\delta_{(\xi, \eta)}$ in the direction of increasing $s$. As in [14], this implies that the probability density $\Psi(\xi, \eta)$ is positive in $E \backslash\left(\gamma_{\theta_{0}} \cup \gamma_{1}\right)$. Then Theorem 2 of [11] (see also [10], [12], [14]) yields the uniqueness of the invariant measure and

$$
\lim _{t \rightarrow \infty}\left\|P_{t} \mu-\Psi\right\|_{L^{1}\left(\mathbb{R}^{2}\right)}=0,
$$

where $\mu_{0}$ is the law of the random variable $\left(\xi_{0}, \eta_{0}\right)$, while $P_{t}$ is the operator defined by

$$
P_{t} \mu_{0}(x, y)=\iint_{\mathbb{R}^{2}} \mu_{0}\left(d x^{\prime}, d y^{\prime}\right) p\left(t, x^{\prime}, y^{\prime}, x, y\right)
$$

This completes the proof of the theorem.

Acknowledgments. The authors wish to thank Prof. Hisao Fujita Yashima of the University of Torino for his suggestions that have been essential for the present work, and the referee for useful comments.

\section{References}

[1] R. M. Anderson and R. M. May, Infectious Diseases of Humans, Oxford Univ. Press, 1991.

[2] P. Baldi, Equazioni differenziali stocastiche e applicazioni, Pitagora, Bologna, 1984.

[3] N. Dalal, D. Greenhalgh and X. Mao, A stochastic model of AIDS and condom use, J. Math. Anal. Appl. 325 (2007), 36-53.

[4] A. Friedman, Stochastic Differential Equations and Applications, Academic Press, 1975 .

[5] T. C. Gard, Introduction to Stochastic Differential Equations, Dekker, New York, 1988.

[6] I. I. Gihman and A. V. Skorohod, Stochastic Differential Equations, Springer, Berlin, 1972 . 
[7] R. Z. Has'minskij, Stochastic Stability of Differential Equations, Sijthoof \& Noordhoof, Alphen aan den Rijn, 1980.

[8] K. Ichihara and H. Kunita, A classification of the second order degenerate elliptic operators and its probabilistic characterization, Z. Wahrsch. Verw. Gebiete 30 (1974), 235-254.

[9] X. Mao, G. Marion and E. Renshaw, Environmental Brownian noise suppresses explosions in population dynamic, Stochastic Process. Appl. 97 (2002), 95-110.

[10] K. Pichór and R. Rudnicki, Stability of Markov semigroups and applications to parabolic systems, J. Math. Anal. Appl. 215 (1997), 56-74.

[11] - - - Continuous Markov semigroups and stability of transport equations, ibid. 249 (2000), 668-685.

[12] R. Rudnicki, On asymptotic stability and sweeping for Markov operators, Bull. Polish Acad. Sci. Math. 43 (1995), 245-265.

[13] - , Long-time behavior of a prey-predator model, Stochastic Process. Appl. 108 (2003), 93-107.

[14] R. Rudnicki, K. Pichór and M. Tyran-Kamińska, Markov semigroups and their applications, in: Lecture Notes in Phys. 597, Springer, Berlin, 2002, 215-238.

[15] E. Tornatore, S. M. Buccellato and P. Vetro, Stability of a stochastic SIR system, Phys. A 354 (2005), 111-126.

[16] E. Tornatore, L. Manca and H. Fujita Yashima, Comportamento asintotico della soluzione del sistema di equazioni stocastiche per due specie in competizione, Istituto Lombardo Accad. Sci. Lett. Rend. A 136/137 (2002/2003), 151-183.

Elisabetta Tornatore

Dipartimento di Matematica ed Applicazioni

Facoltà di Ingegneria, Università degli Studi di Palermo

Viale delle Scienze, 90100 Palermo, Italy

E-mail: elisa@math.unipa.it

Stefania Maria Buccellato

Dipartimento di Matematica ed Applicazioni

Facoltà di Scienze, Università degli Studi di Palermo

Via Archirafi, 34, 90123 Palermo, Italy

E-mail: bucci@math.unipa.it

Received on 12.3.2007;

revised version on 25.10.2007 